\title{
Meeting patients where they are: improving outcomes in early chronic kidney disease with tailored self- management support (the CKD-SMS study)
}

Kathryn Havas ${ }^{1,2^{*}}$, Clint Douglas ${ }^{1}$ and Ann Bonner ${ }^{1,2,3}$

\begin{abstract}
Background: To achieve optimal health outcomes, people with chronic kidney disease must make changes in their everyday lives to self-manage their condition. This can be challenging, and there is a need for self-management support interventions which assist people to become successful self-managers. While interventions have been developed, the literature in this area is sparse and limited by lack of both individualisation and sound theoretical basis. The aim of this study was to implement and evaluate the Chronic Kidney Disease-Self-Management Support intervention: a theory-based, person-centred self-management intervention for people with chronic kidney disease stages 1-4.
\end{abstract}

Methods: A single-sample, pre-post study of an individualised, 12-week intervention based upon principles of social-cognitive theory and person-centred care was conducted with patients attending outpatient renal clinics in Queensland, Australia $(N=66)$. Data were collected at T0 (pre-intervention) and T1 (post-intervention). Primary outcomes were self-efficacy and self-management behaviour.

Results: There were significant, small-to-medium improvements in primary outcomes (self-efficacy: mean difference $+0.8,95 \% \mathrm{Cl} 0.3-1.2, d=0.4$; self-management behaviour: mean difference $+6.2,95 \% \mathrm{Cl} 4.5-7.9, d=0.8)$. There were further significant improvements in secondary outcomes (blood pressure, disease-specific knowledge, physical activity, fruit and vegetable consumption, alcohol consumption, health-related quality of life, psychological distress, and communication with healthcare providers), with effect sizes ranging from negligible to large (all ps <.05).

Conclusions: Social-cognitive theory shows promise as a framework for providing effective person-centred selfmanagement support to patients within this population, and longer-term evaluation is needed.

Trial registration: Australian New Zealand Clinical Trials Registry ACTRN12618000066280. Retrospectively registered 17/01/2018.

Keywords: Chronic kidney disease, Self-management, Self-care, Person-centred care, Patient-centred care, Intervention, Patient education

\footnotetext{
*Correspondence: kathryn.havas@qut.edu.au

'School of Nursing, Queensland University of Technology, Victoria Park Rd,

Kelvin Grove, Brisbane, QLD 4059, Australia

${ }^{2}$ NHMRC Chronic Kidney Disease Centre for Research Excellence, University

of Queensland, St Lucia, Australia

Full list of author information is available at the end of the article
}

(c) The Author(s). 2018 Open Access This article is distributed under the terms of the Creative Commons Attribution 4.0 International License (http://creativecommons.org/licenses/by/4.0/), which permits unrestricted use, distribution, and reproduction in any medium, provided you give appropriate credit to the original author(s) and the source, provide a link to the Creative Commons license, and indicate if changes were made. The Creative Commons Public Domain Dedication waiver (http://creativecommons.org/publicdomain/zero/1.0/) applies to the data made available in this article, unless otherwise stated. 


\section{Background}

Chronic kidney disease (CKD) is a significant burden to those with the disease [1] and healthcare systems [2]. Prevalence is rising, due to increasing rates of diabetes, obesity, and hyptertension [3], and this is predicted to continue [4], with variation across different socioeconomic groups [5]. Burden is particularly high in end-stage kidney disease (ESKD), which requires expensive, time-consuming kidney replacement therapies (KRT; dialysis or transplantation). In earlier stages of CKD, there is an opportunity for interventions to slow progression and improve outcomes. Effective self-management (in areas including diet, physical activity, medication, and smoking and alcohol reduction) impacts health outcomes $[6,7]$; therefore, interventions which improve adherence in these areas have potential to improve outcomes for people with CKD. However, self-management support (SMS) intervention studies for people with CKD, especially earlier stages, are rare and limited by methodological and reporting issues [8-10]. Furthermore, difficulties have been identified in recruiting $[10,11]$ and sustaining $[12,13]$ participation. The current study builds upon the extant literature to address some of these problems, drawing on patient preferences for SMS and a theoretical framework for behavioural change. The personalised intervention reported here (the Chronic Kidney Disease SelfManagement Support program - CKD-SMS) aimed to meet patients where they are, developing goals and tailoring support to improve knowledge, skills, and confidence in self-managing CKD in ways that are congruent with their current knowledge, skills, and engagement with regard to managing their health.
Despite the importance of theory-driven intervention [14], efforts to improve CKD self-management have been largely atheoretical [8]. Additionally, not all behaviour change theories are suited to guide SMS. Some fail to account for the role of emotional states, assuming instead that all behaviour has purely a rational basis $[15,16]$. One effective way of improving CKD self-management is by improving self-efficacy (confidence in ability [17]) to manage the disease $[18,19]$. Social-cognitive theory (SCT [17]) provides a framework for mechanisms of change in a self-efficacy-based model of behaviour. Within a SCT framework (see Fig. 1), self-efficacy is at the heart of behaviour change, and amount of change in behavioural, psychological, and clinical outcomes depends on factors including baseline knowledge and skills, past experiences self-managing, and available coping resources.

Attempts have been made to deliver SCT-based selfmanagement interventions within the CKD literature. However, these have been underdeveloped in attempts to manipulate self-efficacy, tending to focus on only one mechanism of self-efficacy change (including studies focused solely on verbal persuasion, the weakest mechanism [17]), or have not described theoretical reasons for change at all [10]. Furthermore, these studies (like the broader CKD literature) are limited by design and reporting problems [8-10]. A major gap is lack of individualisation, as the CKD population are heterogeneous in terms of support needs and capacity to participate in interventions [20,21]. Additionally, patient activation (engagement with self-management and treatment [22]) in chronic illness is developmental, passing through several stages

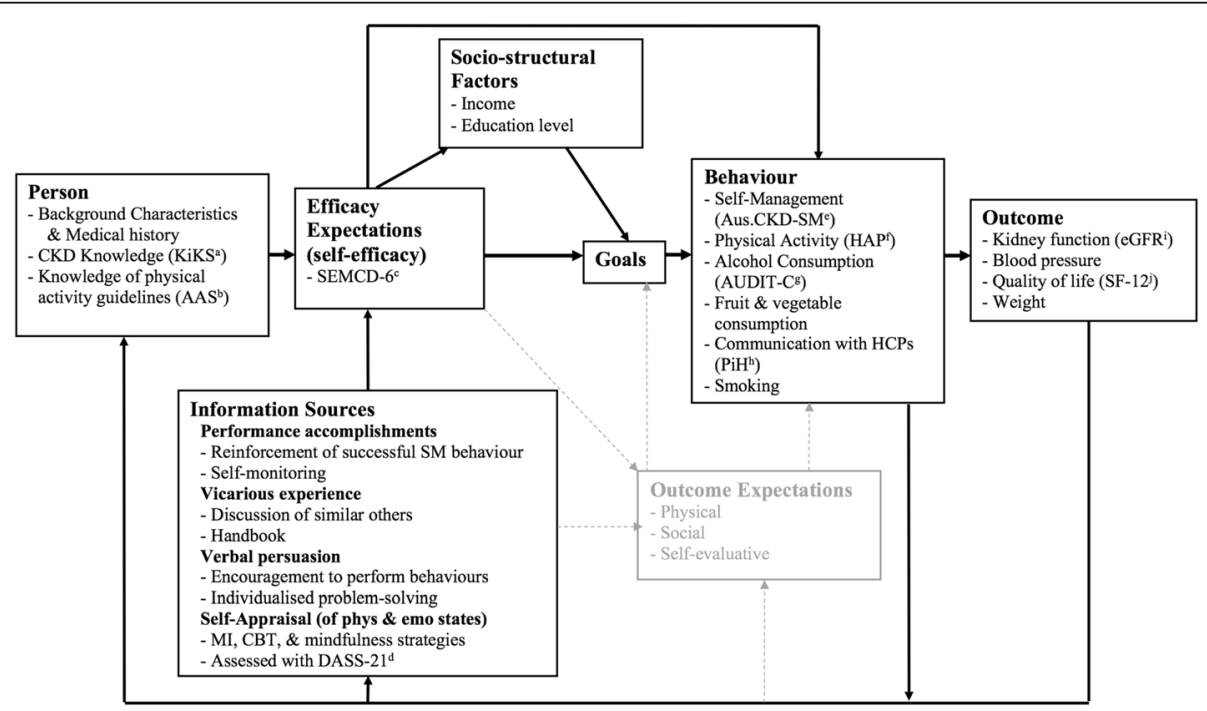

Fig. 1 SCT model (adapted from [19]. 'Kidney Knowledge survey [31]; 'F Four items from the Active Australia Survey [33]; ' Self-Efficacy for Managing Chronic Disease 6-Item Scale [29]; ${ }^{d}$ Depression, Anxiety, and Stress Scales 21-Item Version [32]; ${ }^{\mathrm{C}}$ KKD Self-Management Instrument Australian version [30]; ${ }^{\mathrm{f} H u m a n}$ Activity Profile [35]; ${ }^{9}$ Alcohol Use Disorders Identification Test Consumption Questions Scale [37]; ${ }^{\text {T}}{ }^{\text {wo }}$ items from Partners in Health Scale [36]; 'SF-12v2, Australian version [34] 
(from understanding that participation is important, to ability to maintain effective self-management under stress).

In contrast to a "one-size-fits-all" approach, a personcentred approach [23] to SMS requires an understanding of support desires of the target population and a willingness to take into account individual circumstances, capacity, activation, and motivation while working collaboratively with patients to generate and work towards personally meaningful and attainable goals. People at different stages of activation are likely to benefit from different types of SMS to improve self-efficacy, ranging from basic education to complex problem-solving and skill-development [22]. This exploratory study aimed to evaluate a person-centred, theory-based intervention to improve self-management in people with stage 1-4 CKD (the CKD-SMS). It was hypothesised that the CKD-SMS would lead to increased self-efficacy, CKD knowledge, and engagement in desirable behaviours (CKD self-management, physical activity, fruit and vegetable consumption, and effective communication with healthcare providers (HCPs)), as well as reduction in emotional distress and undesirable behaviours (smoking and alcohol consumption). According to SCT, this should lead to improvements in CKD outcomes (blood pressure (BP), health-related quality of life (HRQoL), weight, and estimated glomerular filtration rate (eGFR)).

\section{Methods}

\section{Design and participants}

This study was a prospective, single-sample, pre-post design. This design was selected over a randomised-controlled trial (RCT) due to aforementioned known challenges with recruitment and participant retention amongst this population, which are such that recruiting and retaining a sample to adequately power a $2 \times 2$ research design was not feasible. Furthermore, the flexibility that is inherent in a person-centred intervention required flexible appointment and rescheduling options, which are incompatible with a highly controlled RCT design. One-sample, repeated-measures designs are common in this field due to the above reasons (e.g., [24-27]), while those studies which have chosen an RCT design cite their small sample sizes as a weakness [28, 29]. Pre-post designs make valuable contributions to the literature when experimental designs are not feasible due to practical constraints such as these [30]. The Transparent Reporting of Evaluations with Nonrandomized Designs (TREND) statement was used to guide reporting [31], and the Template for Intervention Description and Replication (TIDieR) [32] was used to ensure adequate intervention description. Fifty-six participants were required to have $95 \%$ power to detect a medium effect ( $d$ $=0.50$ [33]) in a paired t-test, assuming a $5 \%$ significance level (two-tailed; calculated using G*Power [34]). These estimates were based upon results of $\mathrm{Su}$ and colleagues [19] who used the same primary outcome (the Self-Efficacy for Managing Chronic Disease 6-Item Scale; SEMCD-6) with a similar population to evaluate a SCTbased intervention. Allowing for approximately $30 \%$ attrition, we recruited 78 participants.

Patients who attended five nephrologists' clinics across two public sector outpatient renal clinics in Queensland, Australia were screened by clinic staff for eligibility. These sites are general nephrology outpatient clinics, where patients are typically referred by their General Practitioner or family physician when their eGFR decreases below $60 \mathrm{~mL} / \mathrm{min} / 1.73 \mathrm{~m}^{2}$ or sometimes earlier in the case of sudden kidney function deterioration. Inclusion criteria were: diagnosis of CKD; eGFR $\geq 25 \mathrm{~mL} /$ $\mathrm{min} / 1.73 \mathrm{~m}^{2}$ (so participants would not have commenced pre-dialysis education); $\geq 18$ years of age; and ability to understand English. Exclusion criteria were: cognitive impairment which would inhibit participation (as determined by clinic staff); inability to be followed up (>60 km from researcher's location); and already receiving extensive CKD SMS through select Queensland clinics known to provide enhanced SMS as part of their renal care. Participant recruitment and participation can be seen in Fig. 2 . Data were collected at T0 (baseline) and T1 (13 weeks; 1 week after intervention completion). Due to financial constraints, blinded data collection was not possible.

\section{Procedure}

Recruitment took place between April and December 2016, with follow-up completion in March 2017. Flyers were sent with appointment letters, and verbal consent was obtained for the researcher to approach when patients attended appointments. A systematic recruitment protocol was implemented whereby all eligible patients were approached consecutively by a male or female researcher and provided study information prior to consenting. Participants commenced study instruments, which took approximately 20-30 min to complete, and finalised these at first intervention appointment. The principal investigator, a researcher in CKD self-management with a background in psychology, delivered intervention sessions over a 12-week period. One week after final intervention session, participants completed study questionnaires again faceto-face with the researcher.

\section{Intervention: The CKD-SMS}

The intervention was highly individualised, based upon principles of person-centred care (PCC) and SCT. Expert input was sought (from nephrologists, renal nurses, academic specialists in CKD, and people with CKD) regarding intervention and resource design. The design of the CKD-SMS was also informed by our previous work investigating SMS desires of people with CKD [20, 21], conducted 


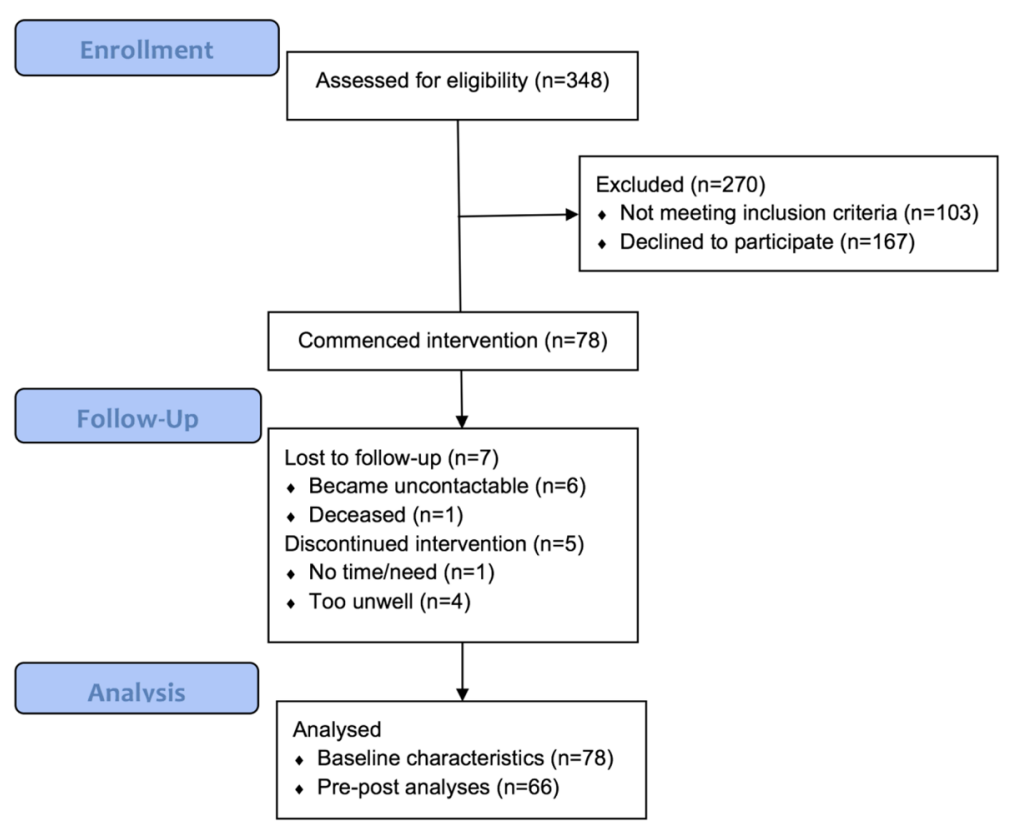

Fig. 2 Participant flow

with the explicit goal of informing intervention development. An outline of the purposes and procedures of CKD-SMS sessions can be seen in Additional file 1.

Participants engaged in two face-to-face intervention sessions with the principal researcher, at week 1 and week 12 , which took place in a mutually convenient location (most often the participant's home or workplace, $80.8 \%$ ). Length of these sessions ranged from 20 to $90 \mathrm{~min}(M=44 \mathrm{~min})$, dependent upon individual need. Between face-to-face sessions, participants nominated preferred frequency of phone sessions (weekly, fortnightly, or monthly), with most (71.2\%) opting for monthly sessions. Phone sessions ranged from five to $60 \mathrm{~min}$, but were mostly brief $(M=12 \mathrm{~min})$. At the first session, participants and researcher collaborated to generate individualised goals in areas including: diet; physical activity; communication with HCPs and engagement with treatment; emotional distress; maintaining roles; and understanding CKD and laboratory results. During the course of the intervention, techniques from SCT (performance accomplishment reinforcement, vicarious experience, and verbal persuasion) were used to assist participants achieve their goals, as were motivational interviewing, cognitive-behavioural, and mindfulness techniques as appropriate (see Additional file 2 for examples of how these strategies were used).

Participants were given a handbook (adapted with permission from Kidney Health Australia's "Living with Reduced Kidney Function" handbook [35]) to accompany the intervention, which was used to prompt discussion. Participants also received self-monitoring and note-taking handouts, ${ }^{1}$ and were encouraged to request further resources. Participants were encouraged to invite family members or friends to attend sessions if desired. One week after the final face-to-face intervention session, participants completed study questionnaires again face-toface with the researcher.

\section{Intervention Fidelity}

All included participants engaged in at least two telephone sessions and two face-to-face sessions during the intervention period. However, during the program, there were 23 instances of participant failure to attend a scheduled face-to-face appointment and 15 instances in which participants were unable to be contacted for scheduled telephone sessions (despite multiple attempts). The personcentred nature of the program meant that flexibility was possible and most participants were able to be followed up and to continue participation in the program.

\section{Outcomes and measurement}

We hypothesised that the CKD-SMS would improve behavioural and patient outcomes, with associated increased knowledge and self-efficacy and reduced emotional distress. Study measures are detailed in Table 1. Primary outcomes were self-efficacy and self-management, assessed using the SEMCD-6 [36] and the Australian version of the Chronic Kidney Disease Self-Management Instrument (Aus.CKDSM) [37], respectively. Secondary outcomes were: HRQoL; CKD knowledge; emotional distress; understanding of physical activity guidelines and engagement in physical activity; fruit and vegetable consumption; communication 


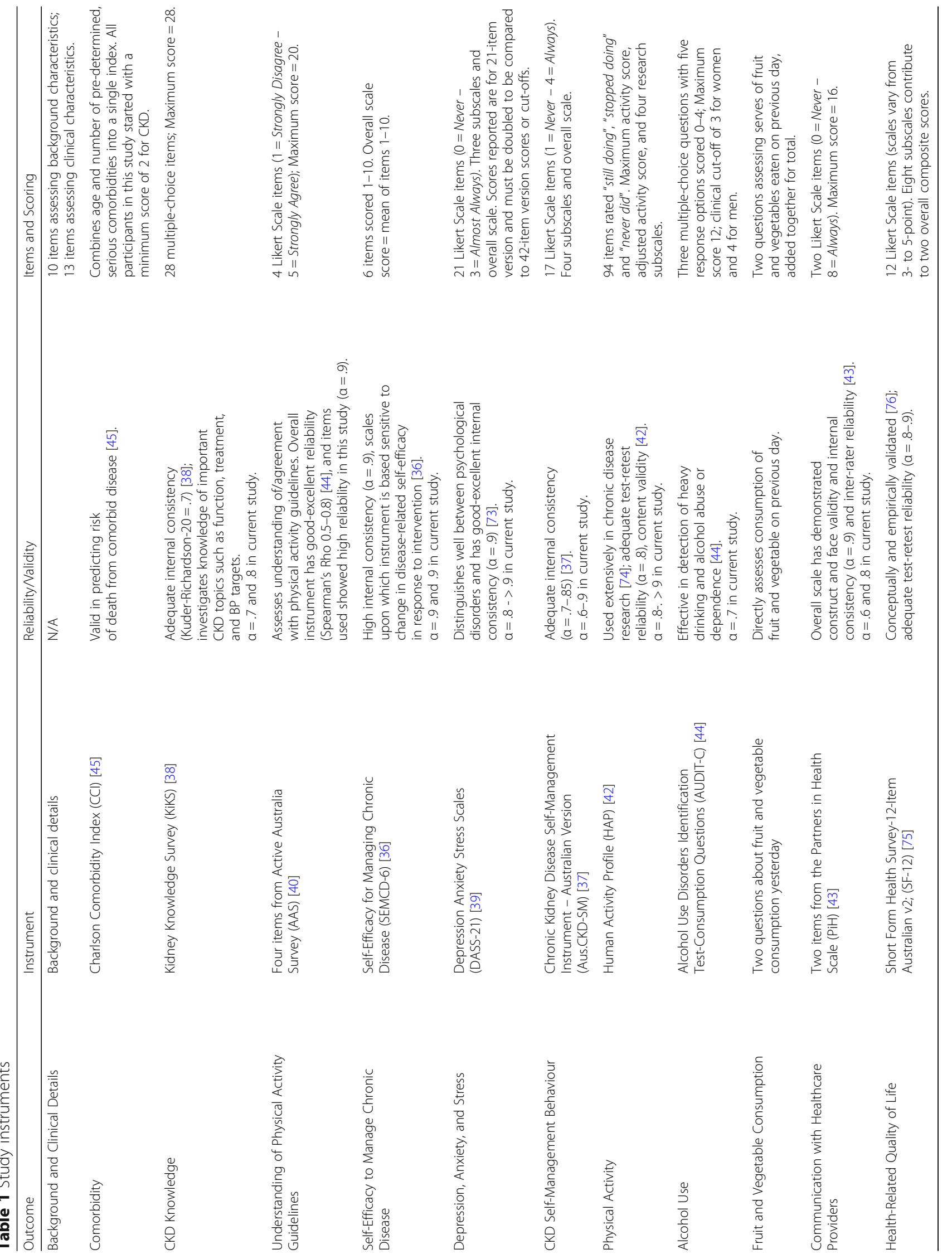


with HCPs; alcohol use; and physiological measures (BP, weight, and eGFR). Additional instruments were: demographic and clinical record form (T0 only); the Kidney Knowledge Survey (KiKS) [38]; the Depression, Anxiety, and Stress Scales 21-Item Version (DASS-21) [39]; four items from the Active Australia Survey (AAS) [40] assessing understanding of physical activity guidelines; the SF-12v2 (Australian version) [41]; the Human Activity Profile (HAP) [42]; two items from the Partners in Health Scale $(\mathrm{PiH})$ [43]; two questions assessing fruit and vegetable consumption; and the Alcohol Use Disorders Test Consumption Questions Scale (AUDIT-C) [44]. Available clinical information at T0 and T1 was gathered from electronic medical records. The Charlson Comorbidity Index (CCI) [45] was used to calculate comorbidity score.

\section{Data analysis}

Data were analysed using IBM SPSS Statistics version 23 [46]. Descriptive statistics were generated for background and clinical data, and T0 and T1 results on patient-reported instruments and clinical measures were compared. Descriptive statistics are presented as frequencies and/or range, mean $(M)$ or median $(M d n)$, and standard deviation (SD) or interquartile range (IQR), as appropriate. Where data met assumptions, paired-samples $t$-tests were conducted to assess change. Where data did not meet assumptions, non-parametric equivalent tests (Wilcoxon Signed-Rank tests) were conducted. Between-groups $t$-tests and Fisher's exact tests were performed to assess for baseline differences between those who completed the intervention and those who did not. Mean differences (Diff) and 95\% confidence intervals (CI), along with effect sizes $(d$ or $r$ ) and statistical significance (at $p<.05)$ are reported for all pre-post-intervention analyses.

\section{Results}

\section{Sample characteristics and participant flow}

Background and clinical characteristics of the original sample $(N=78)$ can be seen in Tables 2 and 3. Slightly over half the sample were female, and age ranged from 25 to 84, with a mean of 57.6. Most participants were born in Australia and predominantly spoke English at home, and two participants identified as Aboriginal or Torres Strait Islander (ATSI). Approximately half (47.4\%) of participants had a high-school or lower level of education and the same number were currently employed. Most had CKD stage 2 or 3, and a third had been living with CKD for 10 years or longer, while some had been diagnosed as recently as 4 months ago.

At baseline, the only difference across background and clinical characteristics and scores on questionnaires between those who completed the intervention and those who did not was that non-completers reported more effective communication with HCPs $(p=.04)$.

\section{Intervention outcomes}

All participants displayed improvement in one or more outcomes between T0 and T1. Details of overall group pre-post intervention changes can be seen in Table 4. Participants identified one or more goals, the most common being overall self-management support (identified 30 times) and knowledge (identified 22 times). The highest percentage of improvement in identified areas was attained for those who set goals of improving knowledge $(86.4 \%)$ or overall self-management $(80.0 \%)$.

\section{Self-efficacy and self-management behaviour}

Comparison of T0 and T1 SEMCD-6 mean scores revealed that participants' self-efficacy to manage CKD improved significantly ( $\mathrm{Diff}=0.8, \mathrm{CI}=0.3-1.2, p=.001$ ), though the effect size was small $(d=0.4)$. Scores on the Aus.CKD-SM indicated that engagement in CKD selfmanagement behaviours also increased during the study period, with significant improvement in scores on the overall instrument (Diff $=6.2, \mathrm{CI}=4.5-7.9, p<.001$ ) and all individual subscales (self-integration; seeking support; adherence to lifestyle modifications; and problem-solving; all $p \mathrm{~s}<.001$ ), with effects ranging from small (for seeking support; $d=0.4$ ) to medium (for all other subscales and overall instrument, $d$ s ranging from 0.5 to 0.8 ). Almost all participants $(63 ; 95.5 \%)$ displayed improvement in one or both primary outcomes.

\section{CKD knowledge}

Change in mean scores on the KiKS revealed significant improvement in CKD knowledge over the course of the intervention $(p<.001)$, with a large effect size $(d=0.8)$.

\section{Knowledge of physical activity guidelines and engagement in physical activity}

Understanding of physical activity guidelines increased significantly over the study period $(p=.02)$, with a small effect size $(d=0.4)$. Similarly, mean scores on the HAP indicated engagement in physical activity also increased. At T1, participants were engaging in more strenuous activities than they were at T0 $(p=.01)$, and more physical activity overall $(p=.02)$, though effect sizes were negligible $(d s=0.2)$, with improvements concentrated in entertainment/social and independent exercise domains.

\section{Health-related quality of life}

Participants demonstrated significant improvement in physical aspects of HRQoL over the study period. Overall physical wellbeing (PCS) improved significantly ( $p$ $=.01)$, with a small effect size $(d=0.30)$, while there was 
Table 2 Background characteristics

\begin{tabular}{|c|c|}
\hline Variable & Frequency $(0$ \\
\hline \multicolumn{2}{|l|}{ Gender } \\
\hline Male & $31(39.7)$ \\
\hline Female & $47(60.3)$ \\
\hline \multicolumn{2}{|l|}{ Age Range: $25-84 \mathrm{M}=57.6 \mathrm{SD}=16.7$} \\
\hline $25-39$ & $14(17.9)$ \\
\hline $40-59$ & $25(32.1)$ \\
\hline $60-79$ & $33(42.3)$ \\
\hline$\geq 80$ & $6(7.7)$ \\
\hline \multicolumn{2}{|l|}{ Place of Birth } \\
\hline Australia & $54(69.2)$ \\
\hline New Zealand & $4(5.1)$ \\
\hline South-east Asia & $4(5.1)$ \\
\hline Europe & $6(7.7)$ \\
\hline Other & $10(12.8)$ \\
\hline \multicolumn{2}{|l|}{ Main Language } \\
\hline English & 73 (93.6) \\
\hline Other & $5(6.4)$ \\
\hline \multicolumn{2}{|l|}{ ATSI ${ }^{a}$ Status } \\
\hline Identifies as Aboriginal & $2(2.6)$ \\
\hline Identifies as neither ATSI & $76(97.4)$ \\
\hline \multicolumn{2}{|l|}{ Marital Status } \\
\hline Single & $15(19.2)$ \\
\hline Married/Defacto & $50(64.1)$ \\
\hline Divorced & $11(14.1)$ \\
\hline Widowed & $2(2.6)$ \\
\hline \multicolumn{2}{|c|}{ Years of Education Range: $0^{b}-24 M=12.9 S D=3.9$} \\
\hline \multicolumn{2}{|l|}{ Highest Educational Qualification Attained } \\
\hline Less than Grade 10 Equivalent & $8(10.4)$ \\
\hline Grade 10 or Equivalent & $22(28.6)$ \\
\hline Grade 12 or Equivalent & $7(9.1)$ \\
\hline TAFE Qualification/Certificate/Diploma & $21(27.3)$ \\
\hline Undergraduate Degree (Bachelors) & $14(18.2)$ \\
\hline Masters Degree & $3(3.9)$ \\
\hline Doctoral Degree (Including PhD) & $2(2.6)$ \\
\hline \multicolumn{2}{|l|}{ Annual Household Income } \\
\hline$<\$ 20,000$ & $10(12.8)$ \\
\hline$\$ 20,000-\$ 39,999$ & $23(29.5)$ \\
\hline$\$ 40,000-\$ 59,999$ & $8(10.3)$ \\
\hline$\$ 60,000-\$ 79,999$ & $7(9.0)$ \\
\hline$\$ 80,000-\$ 99,999$ & $8(10.3)$ \\
\hline$\$ 100,000-\$ 119,999$ & $9(11.5)$ \\
\hline$\$ 120,000+$ & $6(7.7)$ \\
\hline Don't Know/Would Rather not say & $7(9.0)$ \\
\hline
\end{tabular}

Employment Status
Table 2 Background characteristics (Continued)

\begin{tabular}{ll}
\hline Variable & Frequency (\%) \\
\hline Unemployed & $9(11.5)$ \\
Casual & $2(2.6)$ \\
Part Time & $8(10.3)$ \\
Full Time & $25(32.1)$ \\
Retired & $32(41.0)$ \\
Other (Employed) & $2(2.6)$ \\
\hline
\end{tabular}

${ }^{a}$ ATSI Aboriginal or Torres Strait Islander

${ }^{b}$ One participant reported receiving no formal education during her youth in Southeast Asia

no significant change in mental aspects of HRQoL (MCS; $p=.43, d=0.11)$.

\section{Communication with healthcare providers}

Participants' self-reported communication with HCPs improved significantly between T0 and T1 $(p=.01)$, with a small effect size $(d=0.3)$.

\section{Fruit and vegetable consumption, alcohol use, and smoking}

Fruit and vegetable intake improved significantly over the study period, evidenced by self-reported serves consumed on the day prior to assessment $(p<.001)$, with a large effect size $(d=1.7)$. Alcohol consumption decreased significantly $(p=.01)$, though effect size was negligible $(d=0.1)$. There was no change in cigarettes per day amongst the four smokers.

\section{Emotional distress}

There were significant reductions in overall emotional distress as assessed by the DASS-21 between T0 and T1 $(p<.001)$, with a large effect size $(r=.7)$. These improvements were concentrated in the areas of depression $(p=.03)$ and stress $(p=.01)$, with medium effect sizes for both subscales $(r s=.3)$.

\section{Blood pressure}

T0 and T1 BP data was available for 39 participants. Significant improvement was seen in systolic $(p<.01)$ and diastolic $(p=.02)$ measurements, with medium effect sizes $(d \mathrm{~s}=0.7$ and 0.6 , respectively). Percentage of participants at target $(\leq 120 / 80)$ remained stable $(88.5 \%)$.

\section{Discussion}

Through a person-centred, theory-based approach to SMS, this exploratory study demonstrates improved behavioural and patient outcomes among people with stages 1-4 CKD. As postulated by SCT, this was associated with improvements in knowledge, emotional distress, and self-efficacy. The person-centred, flexible approach meant not only that a wide range of people (including those with physical 
Table 3 Clinical characteristics

\begin{tabular}{|c|c|}
\hline Variable & Frequency (\%) \\
\hline \multicolumn{2}{|l|}{ CKD Stage } \\
\hline 1 & $12(15.6)$ \\
\hline 2 & $22(28.6)$ \\
\hline $3 \mathrm{~A}$ & $15(19.5)$ \\
\hline $3 B$ & $20(26.0)$ \\
\hline 4 & $8(10.4)$ \\
\hline \multicolumn{2}{|c|}{ eGFR Range: $25->90 M=57.5 \mathrm{SD}=22.3$} \\
\hline \multicolumn{2}{|c|}{ Creatinine $\mu \mathrm{mol} / \mathrm{L}$ Range: $48-259 \mathrm{M}=116.3 \mathrm{SD}=45.4$} \\
\hline \multicolumn{2}{|c|}{$\begin{array}{l}\text { Time Since Diagnosis (Self-Reported) Range: } 4 \text { months }-33 \text { years } M d n= \\
5 \text { years } Q R=26.3-120.0 \text { (months) }\end{array}$} \\
\hline$\leq 12$ months & $11(14.5)$ \\
\hline 12 years 1 month -3 years & $13(17.1)$ \\
\hline 3 years, 1 month -5 years & $15(19.7)$ \\
\hline 5 years, 1 month $<10$ years & $12(15.8)$ \\
\hline$\geq 10$ years & $25(32.9)$ \\
\hline Unknown & $2(2.6)$ \\
\hline \multicolumn{2}{|l|}{ Cause of CKD } \\
\hline Renovascular & $13(16.9)$ \\
\hline Glomerulonephritis & $13(16.9)$ \\
\hline Diabetes Mellitus (I or II) & $12(15.6)$ \\
\hline Systemic Lupus Erythematosus & $8(10.4)$ \\
\hline Other & $25(32.5)$ \\
\hline Unknown & $6(7.8)$ \\
\hline \multicolumn{2}{|c|}{ Charlson Comorbidity Index Score Range: 2-11 Mdn = 5.5 IQR = 3.3-8.0 } \\
\hline $2-5$ & $36(50.0)$ \\
\hline $6-9$ & $31(43.1)$ \\
\hline $10+$ & $5(6.9)$ \\
\hline \multicolumn{2}{|l|}{ Smoking Status } \\
\hline Non-smoker & $43(55.1)$ \\
\hline Ex-smoker & $28(35.9)$ \\
\hline Current Smoker & $7(9.0)$ \\
\hline \multicolumn{2}{|c|}{ Current Medications Range: 1-14 Mdn: 6} \\
\hline
\end{tabular}

${ }^{\mathrm{a} C K D}$-EPI Creatinine Equation [77]

disabilities such as quadriplegia, blindness, and limb amputation and those with English as their second language, as well as full-time workers with busy schedules) were able to participate, but also that personally meaningful goals were able to be worked towards in order to achieve overall improvements in self-efficacy and self-management. PCC as standard in SMS would mean support was directed where it was needed, a more efficient use of time and resources. The heart failure (HF) literature provides support for this idea, with studies demonstrating that, when delivered as intended, PCC improves patient outcomes and decreases disease burden $[47,48]$.
Individuals are experts on their lives, yet people with chronic disease often do not feel that HCPs value their knowledge and insight regarding their condition [49]. In this study, those with lower self-efficacy at baseline chose more intensive intervention schedules, demonstrating awareness of need for support. SMS in CKD has historically been delivered and evaluated from the perspective of HCPs, focusing on what they know to be important and assuming that provision of disease-specific information will lead to improved self-management [8, 50-52], while failing to account for the complexity of chronic disease self-management from the perspective of their patients. Multiple reviews of CKD self-management intervention studies have been published in recent years $[8-10,53]$, however, they consistently reach similar conclusions: that studies are limited and difficult to review due to large variation in samples, methodologies, and outcomes. In chronic disease, the burden of disease-management is overwhelmingly with the individual, and it is crucial that support processes are in place to set up and maintain effective self-management. In the field of SMS for these diseases, meaningful change for individuals is what is important, and flexibility in intervention and evaluation protocols is going to be necessary in order for this support to reach those who need it most. In contrast to repeated attempts to synthesise overall results of studies in this field, meta-analyses of individual patient data provide techniques which can help to ascertain what works for whom, and under what conditions, rather than continually trying to synthesise disparate studies [54].

After receiving the individualised CKD-SMS, participants displayed overall improvements on several outcomes. Both primary outcomes improved significantly, with small to medium effect sizes. We also found significant improvement in several secondary outcomes. The pattern of findings is consistent with a SCT model of CKD self-management $[17,55,56]$, indicating that the intervention led to changes in knowledge and self-efficacy via multiple sources (education, performance accomplishments, vicarious experience, verbal persuasion, and self-appraisal) which led to changes in behaviour and outcomes. Testing of our SCT model was beyond the scope of this study, but it has provided a framework for future research by proposing a model for self-management in CKD which is empirically testable using standardised measures. In addition to elements assessed in this study, several participants desired assistance with sleep (i.e., training in sleep hygiene), and this was provided during intervention sessions and by way of additional resources. This may be an area that is important to include as an outcome in future studies.

Inclusion of goal-setting and awareness of general SMS needs in planning and implementing person-centred 
Table 4 Pre-post changes in primary and secondary outcomes

\begin{tabular}{|c|c|c|c|c|c|}
\hline & Time - Mean & & & & \\
\hline & Baseline & 12 weeks & Diff $(95 \% \mathrm{Cl})$ & $d$ & $p$ \\
\hline Person Variables & & & & & \\
\hline KiKS & $17.0(5.0)$ & $20.7(3.8)$ & $3.7(2.7-4.8)$ & 0.8 & $<.001$ \\
\hline Understanding of Physical Activity Guidelines & $16.4(2.5)$ & $17.2(2.3)$ & $0.9(0.1-1.6)$ & 0.4 & .02 \\
\hline Self-Efficacy & & & & & \\
\hline SEMCD-6 & $6.7(2.1)$ & $7.44(1.9)$ & $0.8(0.3-1.2)$ & 0.4 & $<.01$ \\
\hline Emotional Distress/Self-Appraisal & & & & & \\
\hline DASS-21 & & & & & \\
\hline Depression $^{\text {ab }}$ & $4.2^{c}\left(4.1^{\mathrm{d}}\right)$ & $3.0^{c}\left(3.3^{\mathrm{d}}\right)$ & $1.2(0.1-2.1)$ & $0.3^{\mathrm{e}}$ & .03 \\
\hline Anxiety $^{\mathrm{ab}}$ & $4.6^{c}\left(4.1^{d}\right)$ & $4.4^{c}\left(4.1^{d}\right)$ & $0.2(-0.91-1.3)$ & $0.1^{\mathrm{e}}$ & .70 \\
\hline Stress $^{\mathrm{ab}}$ & $5.7^{c}\left(4.2^{d}\right)$ & $4.5^{c}\left(3.6^{d}\right)$ & $1.2(0.3-2.1)$ & $0.3^{e}$ & .01 \\
\hline Behaviour & & & & & \\
\hline Aus.CKD-SM & $47.0(8.7)$ & $53.2(7.5)$ & $6.2(4.5-7.9)$ & 0.8 & $<.001$ \\
\hline Self-Integration & $13.7(3.3)$ & $15.8(2.9)$ & $2.2(1.5-2.9)$ & 0.7 & $<.001$ \\
\hline Seeking Support & $8.3(2.7)$ & $9.2(2.7)$ & $1.0(0.4-1.5)$ & 0.4 & $<.001$ \\
\hline Adherence to Lifestyle Modifications & $12.4(2.8)$ & $13.7(2.7)$ & $1.4(0.8-2.0)$ & 0.5 & $<.001$ \\
\hline Problem-Solving & $12.7(2.7)$ & $14.4(2.1)$ & $1.7(1.1-2.3)$ & 0.7 & $<.001$ \\
\hline HAP & & & & & \\
\hline Maximum Activity Score & $69.2(15.6)$ & $72.0(14.8)$ & $2.8(0.6-5.0)$ & 0.2 & .01 \\
\hline Adjusted Activity Score & $59.4(21.5)$ & $62.4(19.3)$ & $3.0(0.6-5.3)$ & 0.2 & .02 \\
\hline Self-care & $7.5(1.6)$ & $7.5(1.5)$ & $<0.1(-0.2-0.2)$ & $<0.1$ & .89 \\
\hline Personal/Household Work & $18.7(6.6)$ & $19.4(5.9)$ & $0.7(-<0.1-1.5)$ & 0.1 & .06 \\
\hline Entertainment/Social & $9.5(2.8)$ & $10.0(2.6)$ & $0.5(<0.1-0.9)$ & 0.2 & .04 \\
\hline Independent Exercise & $8.0(6.5)$ & $9.2(6.8)$ & $1.2(0.1-2.2)$ & 0.2 & .04 \\
\hline AUDIT-C & $2.4(2.7)$ & $2.0(2.3)$ & $0.3(0.1-0.6)$ & 0.1 & .01 \\
\hline Fruit and Vegetables Consumed Yesterday ${ }^{\mathrm{a}}$ & $2.3(1.5)$ & $6.3(3.0)$ & $4.0(3.3-4.8)$ & 1.7 & $<.001$ \\
\hline Communication with $\mathrm{HCPs}^{f}$ & $13.4(2.9)$ & $14.3(2.3)$ & $0.7(0.2-1.6)$ & 0.3 & .01 \\
\hline Cigarettes per day $(n=4)$ & $16.3(4.8)$ & $16.3(4.8)$ & $0.0(-6.5-6.5)$ & $<0.001$ & .99 \\
\hline Outcomes & & & & & \\
\hline eGFR $(n=46)$ & $55.2(24.1)$ & $51.3(24.2)$ & $3.9(1.0-6.9)$ & 0.7 & .01 \\
\hline $\mathrm{BP}(n=39) 88.5 \%$ at target (T0 and T1) & & & & & \\
\hline Systolic & $129.6(23.3)$ & $120.7(15.8)$ & $8.9(2.9-14.9)$ & 0.7 & $<.01$ \\
\hline Diastolic & $74.8(11.1)$ & $70.5(9.6)$ & $4.3(0.9-7.8)$ & 0.6 & .01 \\
\hline SF12 & & & & & \\
\hline PCS & $41.3(11.1)$ & $44.5(8.7)$ & $3.2(0.9-5.4)$ & 0.3 & .01 \\
\hline MCS & $51.9(9.8)$ & $50.9(9.6)$ & $-1.1(-3.8-1.6)$ & 0.1 & .43 \\
\hline Weight $(n=37)$ & $87.4(30.6)$ & $87.8(30.6)$ & $-0.4(-1.1-0.4)$ & 0.3 & .32 \\
\hline
\end{tabular}

$S D$ standard deviation, Diff mean difference, $\mathrm{Cl}$ confidence interval

$d=$ Effect size (small $\geq 0.2$; medium $\geq 0.5$; large $\geq 0.8[40]$ )

${ }^{a} n=56$

bilcoxon Signed-Rank test results reported to deal with effects of violations of t-test assumptions

${ }^{\mathrm{C}}$ Median

${ }^{\mathrm{d}} \mathrm{QQR}$

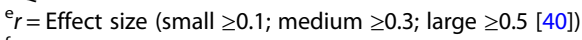

$f^{f}=65$ 
intervention is crucial, as it has been identified that outcome measures often do not even match goals of people with chronic diseases [57]. On an individual, goal-focused level, the areas of greatest improvement were for knowledge and overall self-management. That said, all participants demonstrated improvement in one or more outcomes, indicating that person-centred intervention has the potential to improve patient outcomes overall. This is consistent with previous research across various chronic diseases, which has determined that interventions that are tailored to patient activation [58], preferences [47], values and goals [59], and/or individual circumstances [60] and aligned with principles of PCC [61] can lead to improved patient outcomes. Additionally, HF research has indicated that compatibility of prescribed self-management tasks with life goals is unsurprisingly associated with adherence [62].

The findings of this study are consistent with those of previous research which have indicated that self-management interventions can lead to improved outcomes (see [810, 53] for reviews and limitations). Improvements in BP provide physiological evidence of participant-reported behaviour change, although, as renal function deteriorates, more pharmacological intervention to control BP is required. Smoking behaviour did not improve, however only four smokers participated, and each indicated an unwillingness quit. Smoking cessation is challenging, requiring both readiness and often intensive, targeted intervention [63]. Despite positive outcomes, kidney function declined during the study, although there are multiple possible explanations for this. First, it must be noted that T1 eGFR was only available for participants who were required to see their nephrologist at least every 3 months $(n=46)$, indicating faster kidney function decline. Second, effect from lifestyle modifications takes significant time - longer than the three-month follow-up in this study [64]. Third, understanding about those who are likely to experience CKD progression is emerging, and interventions to slow decline warrant further research.

This research indicates that delivery of individualised, person-centred, theory-based self-management support has potential to help patients with CKD to achieve clinical targets and better health and quality of life outcomes. While our focus was the development of a person-centred intervention, future implementation research is needed to examine its scalability in clinical practice. Elsewhere we report on participants' perspectives of the CKD-SMS intervention, which support this approach as highly useful and helpful in managing their CKD [65]. Yet we acknowledge the systems barriers to change that reduce uptake of person-centred innovation in healthcare [66]. There are opportunities for this intervention to be delivered at existing nurse-led CKD clinics (e.g., [67]), or by nurses alongside routine clinical appointments at outpatient clinics. There is also potential for practices such as this to attract additional funding, with the current international focus on PCC in healthcare practice $[68,69]$. Self-management support desires of people with CKD identified by our previous work [20, 21] could also be used in healthcare contexts to guide goal-setting and development over time. Those with poorer relationships with their HCPs and greater complexity in terms of treatment regimens and comorbidities are likely to need more support than those who feel supported by their HCPs and/or have less complex health problems. People with CKD have to live and manage their condition in an environment fraught with illness and treatment complexity and inconsistency. Person-centred care provides an opportunity to support patients within their complex healthcare environment, and findings from the HF literature demonstrating that PCC helps alleviate feelings of illness-related complexity and ambiguity indicate that it can be effective in doing so [70]. Recognition that people with chronic disease frequently suffer from multiple comorbidities (increasing complexity and rendering advice focused on one discrete illness unhelpful [71]) is also crucial. Government bodies are starting to recognise the importance of integrated care, and to provide subsidies for a HCP in a patient advocate role who has a holistic understanding of individual patients [72].

This study was limited by the fact that data were collected by the researcher who delivered the intervention, which may have encouraged response bias. Selection bias is also possible due to the study design, and may have favoured patients who were motivated to engage in self-management. We were unable to report data on non-consenting patients, however we would expect the intervention effects to be even greater in a more diverse sample. A further limitation of the study was its pre-post design, which does not allow for comparison to an active control group (e.g., one in which participants receive pure information with no SCT or PCC elements). There is an opportunity for future research to build on this study, using longer term evaluations of programs such as the CKD-SMS to assess maintenance of behaviour change and effects on clinical outcomes and disease progression, and also to investigate effects of an intervention such as this in comparison to an active control group. Other constructs not captured in this study such as social support may also be built into the SCT model proposed here.

\section{Conclusions}

CKD research has generally proceeded in ways that ignore the complexity of self-managing chronic illness - both in the design of interventions and the reporting of study outcomes. In doing so, it has fallen short of developing models that are meaningful to people with CKD, and failed to 
provide practitioners with the kind of knowledge needed to best support patients. There are several systematic reviews that are inconclusive or do not provide information about how to optimise SMS to meet patient needs. This study is an important step in moving the field forward: demonstrating improved outcomes in early stage CKD by adopting a person-centred approach to SMS. It also supports SCT as a useful framework to guide future interventions. People with CKD have diverse needs and associated complex comorbidity. It is important to consider individual circumstances, needs and goals, as well as current level of activation, when providing SMS to this population.

\section{Endnotes}

${ }^{1}$ Handbook and handouts available from lead author upon request.

\section{Additional files}

Additional file 1: CKD-SMS 12-Week Program Procedure. (PDF 60 kb) Additional file 2: Clinical Examples of CKD-SMS Strategies. (PDF 58 kb)

\section{Abbreviations}

BP: Blood pressure; CKD: Chronic kidney disease; CKD-SMS: The chronic kidney disease self-management support intervention; ESKD: End-stage kidney disease; HF: Heart failure; KRT: Kidney replacement therapy; PCC: Person-centred care; SCT: Social-cognitive theory

\section{Acknowledgements}

The authors acknowledge the support of the staff at Queensland Kidney Health Services sites and at Kidney Health Australia in developing and conducting this research and the assistance of Vincent Tam as a recruitment and data collection research assistant.

\section{Funding}

This work was supported by the Australian Government and the Queensland University of Technology in the form of an Australian Postgraduate Award, and by the National Health and Medical Research Council Chronic Kidney Disease Centre for Research Excellence by way of a supervisor scholarship. These funding bodies had no involvement in study design; collection, analysis, or interpretation of data; the writing of the report; or in the decision to submit the article for publication.

\section{Availability of data and materials}

The datasets used and/or analysed during the current study are available from the corresponding author on reasonable request.

\section{Authors' contributions}

Conception and design: $\mathrm{KH}, \mathrm{AB}, \mathrm{CD}$; data collection and intervention delivery: $\mathrm{KH}$; data analysis and interpretation: $\mathrm{KH}, \mathrm{AB}, \mathrm{CD}$; manuscript drafting and revisions: $K H, A B, C D$. All authors have read and approved the final manuscript. $\mathrm{KH}$ will act as overall guarantor for the manuscript.

\section{Ethics approval and consent to participate}

This study was approved by the Royal Brisbane and Women's Hospital (EC00172) and Queensland University of Technology (EC00171) Human Research Ethics Committees (approval numbers HREC/15/QRBW/500 and $1,500,001,133$, respectively). Written informed consent was obtained from all participants included in the study.

\section{Consent for publication}

Not applicable.

\section{Competing interests}

The authors declare that they have no competing interests.

\section{Publisher's Note}

Springer Nature remains neutral with regard to jurisdictional claims in published maps and institutional affiliations.

\section{Author details}

${ }^{1}$ School of Nursing, Queensland University of Technology, Victoria Park Rd, Kelvin Grove, Brisbane, QLD 4059, Australia. ${ }^{2}$ NHMRC Chronic Kidney Disease Centre for Research Excellence, University of Queensland, St Lucia, Australia. ${ }^{3}$ Visiting Research Fellow, Kidney Health Service, Metro North Hospital and Health Service, Brisbane, Australia.

Received: 3 October 2017 Accepted: 5 October 2018

Published online: 20 October 2018

\section{References}

1. Almutary H, Bonner A, Douglas C. Symptom burden in chronic kidney disease: a review of recent literature. J Ren Care. 2013;39(3):140-50.

2. World Health Organization. Disease burden by cause, age, sex, by Country and by Region, 2000-2015. Geneva: WHO; 2016.

3. Levin A, Tonelli M, Bonventre J, Coresh J, Donner J-A, Fogo AB, Fox CS, Gansevoort RT, Heerspink HJL, Jardine M, et al. Global kidney health 2017 and beyond: a roadmap for closing gaps in care, research, and policy. Lancet. 2017;390:1888-917.

4. World Health Organization. Projections of mortality and causes of death, 2015 and 2030. Geneva: WHO; 2013.

5. Webster AC, Nagler EV, Morton RL, Masson P. Chronic kidney disease. Lancet. 2017:389(10075):1238-52.

6. Devins GM, Mendelssohn DC, Barre PE, Binik YM. Predialysis psychoeducational intervention and coping styles influence time to dialysis in chronic kidney disease. Am J Kidney Dis. 2003;42(4):693-703.

7. Stenberg U, Haaland-Overby M, Fredriksen K, Westermann KF, Kvisvik T. A scoping review of the literature on benefits and challenges of participating in patient education programs aimed at promoting self-management for people living with chronic illness. Patient Educ Couns. 2016;99(11):1759-71.

8. Bonner A, Havas K, Douglas C, Thepha T, Bennett P, Clark R. Selfmanagement programmes in stages 1-4 chronic kidney disease: a literature review. J Ren Care. 2014;40(3):194-204.

9. Lee M, Wu S, Hsieh N, Tsai J. Self-management programs on eGFR, depression, and quality of life among patients with chronic kidney disease: a meta-analysis. Asian Nurs Res (Korean Soc Nurs Sci). 2016;10(4):255-62.

10. Welch J, Johnson M, Zimmerman L, Russell CL, Perkins SM, Decker B. Selfmanagement interventions in stages 1 to 4 chronic kidney disease: an integrative review. West J Nurs Res. 2015;37(5):652-78.

11. Jacobson Vann JC, Hawley J, Wegner S, Falk RJ, Harward DH, Kshirsagar AV. Nursing intervention aimed at improving self-management for persons with chronic kidney disease in North Carolina Medicaid: a pilot project. Nephrol Nurs J. 2015;42(3):239

12. Byrne J, Khunti K, Stone M, Farooqi A, Carr S. Feasibility of a structured group education session to improve self-management of blood pressure in people with chronic kidney disease: an open randomised pilot trial. British Med J Open. 2011;1(2):e000381.

13. Williams A, Manias E, Liew D, Gock H, Gorelik A. Working with CALD groups: testing the feasibility of an intervention to improve medication selfmanagement in people with kidney disease, diabetes, and cardiovascular disease. Renal Soc Australas J. 2012;8(2):62-9.

14. Glanz K, Bishop DB. The role of behavioral science theory in development and implementation of public health interventions. Annu Rev Public Health. 2010;31(1):399-418.

15. Fishbein M, Ajzen I. Predicting and changing behavior: the reasoned action approach. New York: Psychology Press; 2010.

16. Janz NK, Becker MH. The health belief model: a decade later. Health Educ Behav. 1984;11(1):1-47.

17. Bandura A. Self-efficacy: toward a unifying theory of behavioral change. Psychol Rev. 1977;84(2):191-215.

18. Aliasgharpour M, Shomali M, Moghaddam MZ, Faghihzadeh S. Effect of a self-efficacy promotion training programme on the body weight changes in patients undergoing haemodialysis. J Ren Care. 2012;38(3):155-61. 
19. Su C, Lu X, Chen W, Wang T. Promoting self-management improves the health status of patients having peritoneal dialysis. J Adv Nurs. 2009;65(7):1381-9.

20. Havas K, Bonner A, Douglas C. Self-management support for people with chronic kidney disease: patient perspectives. J Ren Care. 2016;42(1):7-14.

21. Havas K, Douglas C, Bonner A. Person-centred care in chronic kidney disease: a cross-sectional study of patients' desires for self-management support. BMC Nephrol. 2017a;18(1):17.

22. Hibbard JH, Stockard J, Mahoney ER, Tusler M. Development of the patient activation measure (PAM): conceptualizing and measuring activation in patients and consumers. Health Serv Res. 2004;39(4p1):1005-26.

23. Gerteis M, Edman-Levitan S, Daley J, Delbanco T. Through the patient's eyes: understanding and promoting patient-centered care. San Francisco: Jossey-Bass; 1993

24. Kazawa K, Moriyama M. Effects of a self-management skills-acquisition program on pre-dialysis patients with diabetic nephropathy. Nephrol Nurs J. 2013;40(2):141.

25. Walker R, Marshall M, Polaschek N. A prospective clinical trial of specialist renal nursing in the primary care setting to prevent progression of chronic kidney: a quality improvement report. BMC Fam Pract. 2014;15:155.

26. Campbell KL, Ash S, Bauer JD. The impact of nutrition intervention on quality of life in pre-dialysis chronic kidney disease patients. Clin Nutr. 2008; 27(4):537-44.

27. Yen M, Huang JJ, Teng HL. Education for patients with chronic kidney disease in Taiwan: a prospective repeated measures study. J Clin Nurs. 2008; 17(21):2927-34.

28. Chen S-H, Tsai Y-F, Sun C-Y, Wu IW, Lee C-C, Wu M-S. The impact of selfmanagement support on the progression of chronic kidney disease--a prospective randomized controlled trial. Nephrol Dial Transplant. 2011; 26(11):3560-6.

29. Flesher M, Woo P, Chiu A, Charlebois A, Warburton DE, Leslie B. Selfmanagement and biomedical outcomes of a cooking, and exercise program for patients with chronic kidney disease. J Ren Nutr. 2011;21(2):188-95

30. Watson R, McKenna H, Cowman S, Keady J. Nursing research: designs and methods. London: Elsevier Health Sciences UK; 2008.

31. Des Jarlais DC, Lyles C, Crepaz N, The TREND group. Improving the reporting quality of nonrandomized evaluations of behavioral and public health interventions: the TREND statement. Am J Public Health. 2004;94(3):361-6.

32. Hoffmann TC, Glasziou PP, Boutron I, Milne R, Perera R, Moher D, Altman DG, Barbour V, Macdonald H, Johnston M, et al. Better reporting of interventions: template for intervention description and replication (TIDieR) checklist and guide. BMJ. 2014;348:g1687.

33. Cohen J. Statistical power analysis for the behavioral sciences. 2nd ed Hillsdale: Lawrence Erlbaum Associates; 1988.

34. Faul F, Erdfelder E, Lang A, Buchner A. G*power 3: a flexible statistical power analysis program for the social, behavioral, and biomedical sciences. Behav Res Methods. 2007;39(2):175-91.

35. Kidney Health Australia. Living with reduced kidney function: a handbook for self management of chronic kidney disease. Australia: Kidney Health Australia; 2008.

36. Lorig KR, Sobel DS, Ritter PL, Laurent D, Hobbs M. Effect of a selfmanagement program on patients with chronic disease. Eff Clin Pract : ECP. 2001;4(6):256.

37. Wembenyui C, Bonner A, Douglas C. Examining patients' knowledge about chronic kidney disease in a primary health care setting. Queensland: Renal Society of Australasia 44th annual conference; 2016.

38. Wright JA, Wallston KA, Elasy TA, Ikizler TA, Cavanaugh KL. Development and results of a kidney disease knowledge survey given to patients with CKD. Am J Kidney Dis. 2011;57(3):387-95.

39. Lovibond SH, Lovibond PF. Manual for the depression anxiety stress scales. 2nd ed. Sydney: Psychology Foundation; 1995

40. Australian Institute of Health and Welfare. The active Australia survey: a guide and manual for implementation, analysis and reporting. Canberra: Australian institute of health and welfare; 2003.

41. Ware JE, Kosinski M, Bjorner JB, Turner-Bowker DM, Gandek B, Maruish ME. SF-36v2 health survey: Administration guide for clinical trial investigators. Lincoln: QualityMetric Incorporated; 2008.

42. Fix A, Daughton D. Human activity profile professional manual. Odessa: Psychological Assessment Resources, Inc; 1988.

43. Battersby MW, Ask A, Reece MM, Markwick MJ, Collins JP. The Partners in Health scale: the development and psychometric properties of a generic assessment scale for chronic condition self-management. Aus J Prim Health. 2003:9(3):41-52.

44. Bush K, Kivlahan DR, McDonell MB, Fihn SD, Bradley KA. The AUDIT alcohol consumption questions (AUDIT-C): an effective brief screening test for problem drinking. Ambulatory care quality improvement project (ACQUIP). Alcohol use disorders identification test. Arch Intern Med. 1998;158(16):1789-95.

45. Charlson M, Szatrowski TP, Peterson J, Gold J. Validation of a combined comorbidity index. J Clin Epidemiol. 1994;47(11):1245-51.

46. Corp IBM. SPSS for mac. 23.0 ed. Armonk: IBM Corp; 2015.

47. Brännström M, Boman K. Effects of person-centred and integrated chronic heart failure and palliative home care. PREFER: a randomized controlled study. Eur J Heart Fail. 2014;16(10):1142-51.

48. Hansson E, Ekman I, Swedberg K, Wolf A, Dudas K, Ehlers L, Olsson L-E. Person-centred care for patients with chronic heart failure - a cost-utility analysis. Eur J Cardiovasc Nurs. 2016:15(4):276-84.

49. Zoffmann V, Harder I, Kirkevold M. A person-centered communication and reflection model: sharing decision-making in chronic care. Qual Health Res. 2008;18(5):670-85.

50. Granger BB, Sandelowski M, Tahshjain H, Swedberg K, Ekman I. A qualitative descriptive study of the work of adherence to a chronic heart failure regimen: patient and physician perspectives. J Cardiovasc Nurs. 2009;24(4):308-15.

51. Lake AJ, Staiger PK. Seeking the views of health professionals on translating chronic disease self-management models into practice. Patient Educ Couns. 2010;79(1):62-8.

52. Sadler E, Wolfe CD, McKevitt C. Lay and health care professional understandings of self-management: a systematic review and narrative synthesis. SAGE Open Med. 2014;2(0). https://doi.org/10.1177/2050312114544493.

53. Lin M-Y, Liu MF, Hsua L-F, Tsai P-S. Effects of self-management on chronic kidney disease: a meta-analysis. Int J Nurs Stud. 2017;74:128-37.

54. Jonkman NH, Groenwold RHH, Trappenburg JCA, Hoes AW, Schuurmans MJ. Complex self-management interventions in chronic disease unravelled: a review of lessons learned from an individual patient data meta-analysis. J Clin Epidemiol. 2017:83:48-56.

55. Bandura A. Health promotion by social cognitive means. Health Educ Behav. 2004;31(2):143-64

56. Curtin RB, Walters BAJ, Schatell D, Pennell P, Wise M, Klicko K. Self-efficacy and self-management behaviors in patients with chronic kidney disease. Adv Chronic Kidney Dis. 2008;15(2):191-205.

57. Gardner T, Refshauge K, McAuley J, Goodall S, Hubscher M, Smith L. Patient led goal setting in chronic low back pain-what goals are important to the patient and are they aligned to what we measure? Patient Educ Couns. 2015;98(8):1035-8.

58. Hibbard JH, Greene J, Tusler M. Improving the outcomes of disease management by tailoring care to the patient's level of activation. Am J Manag Care. 2009;15(6):353-60.

59. Lundgren J, Andersson G, Dahlstrom O, Jaarsma T, Kohler AK, Johansson P, Institutionen för medicin och h, Medicinska f, Hjärt- och M, Institutionen för beteendevetenskap och I, et al. Internet-based cognitive behavior therapy for patients with heart failure and depressive symptoms: a proof of concept study. Patient Educ Couns. 2015;98(8):935-42.

60. Lion KC, Mangione-Smith R, Britto MT. Individualized plans of care to improve outcomes among children and adults with chronic illness: a systematic review. Care Manag J. 2014;15(1):11-25.

61. Ekman I, Wolf A, Olsson L-E, Taft C, Dudas K, Schaufelberger M, Swedberg K. Effects of person-centred care in patients with chronic heart failure: the PCC-HF study. Eur Heart J. 2012;33(9):1112-9.

62. Zhang KM, Dindoff K, Arnold JMO, Lane J, Swartzman LC. What matters to patients with heart failure? The influence of non-health-related goals on patient adherence to self-care management. Patient Educ Couns. 2015;98(8):927-34.

63. Australian Government Department of Health and Ageing. Smoking cessation guidelines for Australian general practice. Australia: The Royal Australian College of General Practitioners; 2012.

64. Enworom CD, Tabi M. Evaluation of kidney disease education on clinical outcomes and knowledge of self-management behaviors of patients with chronic kidney disease. Nephrol Nurs J. 2015;42(4):363-73.

65. Havas K, Douglas C, Bonner A. Closing the loop in person-centered care: patient experiences of a chronic kidney disease self-management intervention. Patient Prefer Adherence. 2017:11:1963-73.

66. Brummel-Smith K, Butler D, Frieder M, Gibbs N, Henry M, Koons E, Loggers E, Porock D, Reuben DB, Saliba D, et al. Person-centered care: a definition and essential elements. J Am Geriatr Soc. 2016;64(1):15-8. 
67. Coleman S, Havas K, Ersham S, Stone C, Taylor B, Graham A, Bublitz L, Purtell $\mathrm{L}$, Bonner A. Patient satisfaction with nurse-led chronic kidney disease clinics: a multicentre evaluation. J Ren Care. 2017;43(1):11-20.

68. Australian Commission on Safety and Quality in Healthcare. Patient-centred care: Improving quality and safety through partnerships with patients and consumers. Sydney: Australian Commission on Safety and Quality in Healthcare; 2011.

69. NHS England. Putting patients first - The NHS England business plan for 2013/14-2015/16. London: The Stationery Office; 2013.

70. Dudas K, Olsson L-E, Wolf A, Swedberg K, Taft C, Schaufelberger M, Ekman I. Uncertainty in illness among patients with chronic heart failure is less in person-centred care than in usual care. Eur J Cardiovasc Nurs. 2013;12(6):521-8.

71. Bowling CB, Vandenberg AE, Phillips LS, McClellan WM, Johnson TMI, Echt KV. Older patients' perspectives on managing complexity in CKD selfmanagement. Clin J Am Soc Nephrol. 2017;12:635-43.

72. Bayliss EA, Balasubramianian BA, Gill JM, Stange KC. Perspectives in primary care: implementing patient-centered care coordination for individuals with multiple chronic medical conditions. Ann Fam Med. 2014;12(6):500-3.

73. Antony MM, Bieling PJ, Cox BJ, Enns MW, Swinson RP. Psychometric properties of the 42-item and 21-item versions of the depression anxiety stress scales in clinical groups and a community sample. Psychol Assess. 1998:10(2):176-81.

74. Davidson M, de Morton N. A systematic review of the human activity profile. Clin Rehabil. 2007;21(2):151-62.

75. Ware JE, Kosinski M, Keller SD. A 12-item short-form health survey: construction of scales and preliminary tests of reliability and validity. Med Care. 1996;34(3):220-33.

76. Sanson-Fisher RW, Perkins JJ. Adaptation and validation of the SF-36 health survey for use in Australia. J Clin Epidemiol. 1998;51:961-7.

77. Levey AS, Stevens LA, Schmid CH, Zhang YL, Castro AF 3rd, Feldman HI, Kusek JW, Eggers P, Van Lente F, Greene T, et al. A new equation to estimate glomerular filtration rate. Ann Intern Med. 2009;150(9):604-12.

Ready to submit your research? Choose BMC and benefit from:

- fast, convenient online submission

- thorough peer review by experienced researchers in your field

- rapid publication on acceptance

- support for research data, including large and complex data types

- gold Open Access which fosters wider collaboration and increased citations

- maximum visibility for your research: over $100 \mathrm{M}$ website views per year

At $\mathrm{BMC}$, research is always in progress.

Learn more biomedcentral.com/submissions 